\title{
STRUCTURES AND FUNCTIONS OF OLIGOSACCHARINS
}

Three-Year Renewal

June 15, 1996-June 14, 1999

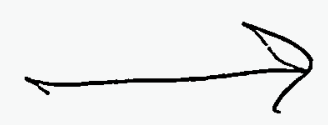

\author{
Progress Report \\ June 15, 1993-June $14,19.96:$ \\ Dr. Peter Albersheim \\ Principal Investigator \\ Complex Carbohydrate Research Center \\ The University of Georgia \\ Athens, Georgia 30602-4712
}

December 1995

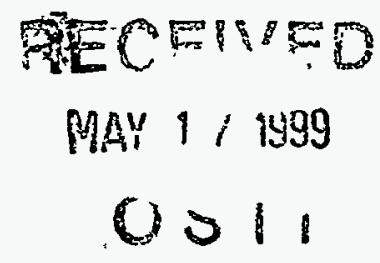

PREPARED FOR THE

U.S. DEPARTMENT OF ENERGY

UNDER GRANT DE-FG05-93ER20114 


\section{DISCLAIMER}

Portions of this document may be illegible in electronic image products. Images are produced from the best available original document. 


\title{
STRUCTURES AND FUNCTIONS OF OLIGOSACCHARINS
}

\author{
Progress Report
}

\section{June 15, 1993-December 31, 1996}

We have made considerable progress during the 2.5 year funding period just ending in our studies of the structures and functions of oligosaccharide signal molecules (oligosaccharins). We have emphasized studies of the enzymes that solubilize, process, and degrade oligosaccharins and of the proteins that inhibit those enzymes. We have been especially interested in elucidating how oligosaccharins and their processing enzymes participate in determining the outcome of challenges to plants by pathogenic microbes. We have studied, to a lesser extent, the roles of oligosaccharins in plant growth and development. Abstracts of papers describing results acquired with support from this grant that have been published, submitted, or in preparation are presented below to summarize the progress made during the last two and one half years. The following paragraphs highlight the most important contributions made in our oligosaccharin research during this time period, and the corresponding abstract is referred to by its assigned number in parentheses. Results of work in progress are described primarily in conjunction with our application for continued support.

The results of the studies supported by this grant have contributed to the realization that the battles between plants and microbes are often waged in the extracellular matrices of the combatants $(5,8)$. We and others have obtained evidence that oligosaccharins, solubilized from wall polysaccharides or glycoproteins of the plant and/or the microbe, appear to play a central role in the battle. When the concentration of a defense-related oligosaccharin accumulates to bioactive levels $\left(10^{-7}\right.$ to $\left.10^{-9} \mathrm{M}\right)$, the plants perceive the signal and respond by activating their defense responses $(5,8)$. One of the contributions of our research was the demonstration that the biochemical response of plants to an infection by a microbe results in an enhanced accumulation of oligosaccharins $(6,11)$, while pathogens, when attempting to infect a plant, utilize biochemical mechanisms that prevent the accumulation of oligosaccharins (10, 12). We hypothesize that the winner of this competition determines whether the interaction between a host and pathogen results in a diseased (compatible interaction) or healthy (incompatible interaction) plant.

One of our oligosaccharin studies centers on the interactions between fungal endopolygalacturonases (EPGs), plant polygalacturonase-inhibiting proteins (PGIPS), and oligogalacturonide oligosaccharins (6): We have established that when a fungal EPG, in the presence of its homogalacturonan substrate, is inhibited by PGIP, oligosaccharin-active oligogalacturonides (those with DPs 12-14) accumulate and activate plant defense responses (6). Our evidence supporting the hypothesis that PGIP is an important defense factor of plants received a great deal of support by the discovery that the nucleotide sequence of bean pgip ( $P$. Toubart, A. Desiderio, G. Salvi, F. Cervone, L. Daroda, and G. De Lorenzo, 1992, Plant J. 2:367-373) is closely related to the sequence of the Cf-9 resistance gene of tomato (D.A. Jones, C.M. Thomas, K.E. Hammond-Kosack, P.J. Balint-Kurti, and J.D.G. Jones, 1994, Science 266:789-793). Further evidence supporting the defense role of PGIP is provided by our demonstration that, although PGIP is present constitutively in bean plants, it is induced to higher levels by fungal-derived glucan elicitor, plant-derived oligogalacturonide elicitor, salicylic acid, and wounding of the plant tissues $(6,11)$; these are all treatments that are known to activate defense responses in plants. We have also shown that PGIP accumulates in those cells immediately surrounding fungal infection sites (11). These results support the hypothesis 
that PGIP is a central component of the defense processes of plants $(6,11)$. Further evidence in support of this hypothesis has been provided by the demonstration that over-expression of pear pgip in tomato fruit provides protection against infection by Botrytis cinerea, a fungal pathogen of tomato (A.L.T. Powell and A. B. Bennett, In Proceedings of the Int'l Plant Molecular Biology Conference, Amsterdam, 1994). We are collaborating with John Bedbrook of United Agricorp (see appended letter) to evaluate the ability of over-expressed bean pgip to protect several crop plants.

Another important discovery of this funding period was the demonstration that at least some pathogenic fungi synthesize proteins that inhibit anti-fungal endoglucanases synthesized by plants. It has long been known that plants produce pathogenesis-related (PR) proteins in response to pathogen attack. Quite a few of the PA proteins have been shown to be endo- $\beta$ 1,3-glucarases and chitinases, which aid in protecting plants from pathogenesis by degrading the structural polysaccharides of mycelial cell walls and by releasing oligosaccharins from mycelial walls that activate plant defenses. We have shown that some fungal pathogens secrete proteins that inhibit plant endo- $\beta-1,3-$ glucanases $(14,15)$. These glucanase-inhibitor proteins (GIPs) appear to be highly selective with regard to the endoglucanases they inhibit (10, 14). We hypothesize that the GIPs protect the pathogen's mycelial walls from being degraded by the plant's endoglucanases and simultaneously minimize the formation from the mycelial walls of glucan oligosaccharins, which the plant uses as a signal to activate defense genes. Further studies of GIPs represent a significant portion of our proposed research.

We have also looked carefully in three host-pathogen systems for inhibitors of PR endochitinases; none were found. However, we did find that some fungi secrete both endoand exochitinases that degrade the oligosaccharin-active chitin oligomers (DPs -4-6) that are released from fungal mycelial walls by the PA endochitinases of the host plant (unpublished results). Thus, the fungal chitinases may prevent the plant from detecting oligosaccharins derived from the fungal mycelial wall.

Our host-pathogen studies have advanced on another front. Our earlier work provided evidence that Magnaporthe grisea, the fungus that causes rice blast disease, secretes an endo$\beta-1,4-x y l a n a s e$ and an $\alpha$ - $L$-arabinofuranosidase that generate oligosaccharin-active arabinoxylan fragments from rice cell walls (9). These results led us to hypothesize that the endo- $\beta-1,4-x y l a n a s e$ is an important pathogenicity factor of the fungus. Therefore, we decided to purify, clone, and characterize not one but two endo- $\beta-1,4-x y l a n a s e s$ from $M$. grisea (9). Using gene replacement technology, we generated $M$. grisea mutants lacking either or both of the endo- $\beta-1,4-x y l a n a s e s$ (see Proposed Research). Of considerable interest is our observation that the double mutant lacking both enzymes still infects compatible rice cultivars and induces the hypersensitive response on incompatible cultivars and non-host plants. We then found that the double mutant secretes endoxylanase activity, even though we had inactivated (by homologous recombination) all of the $M$. grisea genes homologous to known xylanases.

We have made considerable progress in our studies of xyloglucan (XG)-derived oligosaccharins. In a previously funded DOE grant, we identified the structure of a terminal fucose-containing $X G$ nonasaccharide that at nanomolar concentrations inhibits auxinstimulated growth of pea epicotyl segments. In this most recent funding period we studied an $\alpha$-fucosidase that inactivates the XG oligosaccharin. We determined that the enzyme is specific for the XG oligosaccharides that contain fucosyl residues and that production of the enzyme is developmentally regulated (1). We established that the enzyme is localized in the cell wall and 
that this fucosidase may be an important factor in plant growth regulation based on its potential control of the concentration of this growth-regulating oligosaccharin (7).

This same XG-derived, growth-regulating nonasaccharide is central to our studies with Arabidopsis mutant mur1, which is deficient ( $>99 \%$ reduction) in fucosyl residues in the aerial parts of the plant. The mutant goes through an apparently normal life cycle, but the mutant is slightly smaller and more brittle than the parent. It was suggested (W.-D. Reiter, C.S. Chapple, and C.R. Sommerville, 1995, Science 261:1032-1035) that the survival of this mutant places in doubt the importance of the proposed growth-regulating function of the fucose-containing oligosaccharin, as the fuçose is required for the oligosaccharin's bioactivity. Our studies (12) confirmed that the fucosyl-containing nonasaccharide is not present in the mutant, but we discovered that the mutant replaces the $\alpha$-L-fucosyl residue with an $\alpha$-L-galactosyl residue, a sugar that has rarely been observed in higher plants. Galactose has the same stereochemistry as fucose and differs from fucose only in the presence of an oxygen atom attached to C-6. Indeed, terminal $\alpha$-L-galactosyl residues probably adopt the same conformation as terminal $\alpha-L-$ fucosyl residues (12). Therefore, we were not surprised to find that the $X G$-derived nonasaccharide, with L-galactose substituting for L-fucose, has an activity indistinguishable from that of the L-fucose-containing nonnasaccharide in the pea epicotyl growth inhibition assay. Thus, mur1 contains an oligosaccharin-functional XG nonasaccharide subunit, supporting the hypothesis that oligosaccharins are important to growth and development, as we recently reviewed $(5,8)$.

\section{Abstracts of Published Papers}

1. Augur, C., N. Benhamou, A. Darvill, and P. Albersheim. 1993. Purification, characterization, and cell wall localization of an $\alpha$-fucosidase that inactivates a xyloglucan oligosaccharin. Plant J. 3: 415-426.

An $\alpha$-fucosidase that releases fucosyl residues from oligosaccharide fragments of xyloglucan, a plant cell wall hemicellulosic polysaccharide, was purified to homogeneity from pea (Pisum sativum) epicotyls using a combination of cation exchange chromatography and isoelectric focusing. The $\alpha$-fucosidase has a molecular mass of $20 \mathrm{kDa}$ according to sodium dodecyl sulfate-polyacrylamide gel electrophoresis. The $\alpha$-fucosidase has an isoelectric point of 5.5. The substrate specificity of the $\alpha$-fucosidase was determined by high performance anion exchange chromatographic analysis of oligosaccharide substrates and products. The enzyme hydrolyzes the terminal $\alpha-1,2$-fucosidic linkage of oligosaccharides and does not cleave $p$ nitrophenyl- $\alpha-L$-fucoside. The enzyme does not release measurable amounts of fucosyl residues from large polysaccharides. The subcellular localization of $\alpha$-fucosidase in pea stems and leaves has been studied by immunogold cytochemistry. The $\alpha$-fucosidase accumulates in primary cell walls and is not detectable in the middle lamella or in the cytoplasm of 8-day-old stem tissue and 14-day-old leaf tissue. $\alpha$-Fucosidase activity was readily detected in extracts of 8 -day-old stem tissue. No significant $\alpha$-fucosidase activity or immunogold labeling of the $\alpha$ fucosidase was detected in 2- and 4-day-old stem tissue indicating that production of $\alpha$ fucosidase is developmentally regulated.

2. Bellincampi, D., G. Salvi, G. De Lorenzo, F. Cervone, V. Marfà, S. Eberhard, A. Darvill, and $P$. Albersheim. 1993. Oligogalacturonides inhibit the formation of roots on tobacco explants. Plant J. 4: 207-213.

$\alpha-1,4$-Oligogalacturonides with degrees of polymerization (DPs) ranging from 6 to 18 or 2 to 8 were added to tobacco leaf explants, and root formation was evaluated after 15 days of 
incubation. Auxin-induced formation of roots was inhibited by oligogalacturonides with DPs 618 but not by the oligogalacturonides with DPs $2-8$. The inhibition of root formation by the larger oligogalacturonides was prevented by increasing the amount of auxin present in the medium. Oligogalacturonides (DPs 6-18) also inhibited root formation when added to tobacco thin cell-layer (TCL) explants in a medium that is known to induce the formation of roots. The addition of size-homogeneous oligogalacturonides, to either tobacco leaf explants or TCLs, established that oligogalacturonides with DPs between 10 and 14 were most active in inhibiting the formation of roots. These data suggest that oligogalacturonides of the same size as those known to elicit plant defense responses, and to affect floral development and membrane functions, also inhibit the induction of root organogenesis in tobacco.

3. Brady, K.P., A.G. Darvill, and P. Albersheim. 1993. Activation of a tobacco glycinerich protein gene by a fungal glucan preparation. The Plant $J .4:$ 517-524.

A Phytophthora megasperma f.sp. glycinea cell wall glucan preparation was previously shown to protect tobacco plants against viral infection. Eleven plant defense-related genes were assayed for elevated mRNA accumulation levels in response to glucan treatment of tobacco plants. The expression of only one of these genes, a glycine-rich protein (GRP) gene, was induced by glucan application. Elevated GRP gene mRNA levels could be detected within $15 \mathrm{~min}$ of glucan treatment and reached maximum levels at $4 \mathrm{~h}$ post-treatment followed by a slow decline to $8 \mathrm{~h}$. The maximum induction of the GRP gene was approximately ninefold above $\mathrm{H}_{2} \mathrm{O}$-treated control plants. Northern blot analysis showed that a single mRNA species of $1.4 \mathrm{~kb}$ was responding to the glucan treatment. GRP genes occur in tobacco as members of a multigene family, but only one specific GRP gene was induced by the glucan treatment. A genomic copy of this responding GRP gene was cloned and sequenced. This tobacco GRP gene is homologous to the petunia ptGRP1 gene and the French bean GRP1.8 gene, but is not closely related to the French bean GRP1.0 gene. GRP gene expression has previously been associated with disease resistance in plants, but it remains to be determined whether $\beta$-glucan activation of the tobacco GRP gene results in the observed resistance to virus.

4. Caprari, C., C. Bergmann, Q. Migheli, G. Salvi, P. Albersheim, A. Darvill, F. Cervone, and G. De Lorenzo. 1993. Fusarium moniliforme secretes four endopolygalacturonases derived from a single gene product. Physiol. Mol. Plant Path. 43: 453-462.

Extracellular endopolygalacturonase, purified from the pathogenic fungus Fusarium moniliforme, consists of four molecular forms $(38,41.5,45$, and $48.5 \mathrm{kDa}$, respectively). Three forms $(38,41.5$, and $45 \mathrm{kDa})$ were purified to homogeneity by FPLC on a Mono $S$ column followed by electroelution after SDS-PAGE. The $\mathrm{N}$-terminal amino acid sequences of each of the three forms, and of a mixture containing all four forms were shown to be identical to that predicted from the nucleotide sequence of the endopolygalacturonase gene previously cloned from $F$. moniliforme. Enzymatic deglycosylation experiments revealed the presence of $N$ linked, high mannose oligosaccharide side-chains on all four forms of endopolygalacturonase. Hydrogen fluoride catalyzed chemical deglycosylation of the polygalacturonase mixture yielded a single polypeptide with an apparent molecular mass of $36.2 \mathrm{kDa}$. Southern blot analysis, carried out at high stringency with an endopolygalacturonase-specific probe on genomic DNA digested with three different restriction enzymes, showed a single hybridizing restriction fragment in all three digests. A single $2.0 \mathrm{Mb}$ chromosome hybridized with the endopolygalacturonase-speciflc probe, as shown by Southern blot analysis of $F$. moniliforme chromosomes separated by CHEF electrophoresis. Northern blot analysis revealed only one 
mRNA species (1350 nt) encoding endopolygalacturonase. These data indicate that a single gene encodes the endopolygalacturonases of $F$. moniliforme.

5. Albersheim, P., J. An, G. Freshour, M.S. Fuller, R. Guillen, K.-S. Ham, M.G. Hahn, J. Huang, M. O'Neill, A. Whitcombe, M.V. Williams, W.S. York, and A. Darvill. 1994. Structure and function studies of plant cell wall polysaccharides. Blochem. Soc. Trans. 22: 374-378.

The plant cell wall, which is the major source of biomass and dietary fibre, is a vital natural resource. Primary plant cell walls, that is, the walls of growing cells, govern many of the fundamental properties of plant cells. The walls provide the first barrier to pests, they physically control the rate of cell growth, they are the organelle that ultimately controls the shape of plant cells and consequentiy of organs and whole organisms, and they are the source of an important class of regulatory molecules called oligosaccharins. Thus, elucidating the structures and functions of plant cell walls is fundamental to plant science.

Primary cell walls are composed of about $20 \%$ cellulose microfibrils, $70-80 \%$ non-cellulosic polysaccharides, and up to $10 \%$ structural glycoproteins. Structural elucidation of primary cell wall polymers is essential to dissecting and understanding their roles in wall structure and other physiological functions. Although considerable progress has been made in delineating the primary structures of the non-cellulosic cell wall polysaccharides, many questions remain to be answered before a reasonably accurate picture of primary cell walls can be visualized. For example, do all cells contain all of the wall polysaccharides? Do the polysaccharides of the walls of different cells and different tissues have the same or different structures? How are the various polysaccharides distributed in the walls of the cells of different tissues, organs, and species? What are the three-dimensional structures of these polysaccharides? How are the polysaccharides themselves and polysaccharides and structural glycoproteins interconnected? Are all the glycosyl linkages and glycosyl residues of which the wall polysaccharides are composed essential for wall integrity? Can mutants be obtained that lack one or more wall components but have normal or at least minimally functional walls?

Partial answers to these questions are available, and the answers are intriguing. We have, for many years, been impressed by the conservation of the structures of the wall polysaccharides. Cellulose has the same structure in organisms as diverse as bacteria and trees. Three galacturonic acid-rich pectic polysaccharides, thamnogalacturonan (RG)-I, RG-II and homogalacturonan, and two cellulose-binding hemicelluloses, glucuronoarabinoxylan and xyloglucan, are all present in all of the higher plants that have been studied, and their structures, in even the most evolutionarily diverse plants, are sufficiently conserved that there is never a problem of knowing what polysaccharide you are examining. But are their structures the same in all plants and in all cells of the same plant?

6. Bergmann, C.W., Y. Ito, D. Singer, P. Albersheim, A.G. Darvill, N. Benhamou, L. Nuss, G. Salvi, F. Cervone, and G. De Lorenzo. 1994. Polygalacturonase-inhibiting protein accumulates in Phaseolus vulgaris $L$. in response to wounding, elicitors and fungal Infection. Plant J. 5: 625-634.

Polygalacturonase-inhibiting protein (PGIP) is a cell wall-associated protein that specifically binds to and inhibits the activity of fungal endopolygalacturonases. The Phaseolus vulgaris gene encoding PGIP has been cloned and characterized. Using a fragment of the cloned pgip gene as a probe in Northern blot experiments, it is demonstrated that the pgip mRNA accumulates in suspension-cultured bean cells following addition of elicitor-active oligogalacturonides or fungal glucan to the medium. Rabbit polyclonal antibodies specific for 
PGIP were generated against a synthetic peptide designed from the $N$-terminal region of PGIP; the antigenicity of the peptide was enhanced by coupling to KLH. Using the antibodies and the cloned pgip gene fragment as probes in Western and Northern blot experiments, respectively, it is shown that the levels of PGIP and its mRNA are increased in $P$. vulgaris hypocotyls in response to wounding or treatment with salicylic acid. Using gold-labeled goat-anti-rabbit secondary antibodies in EM studies, it has also been demonstrated that, in bean hypocotyls infected with Colletotrichum lindemuthianum, the level of PGIP preferentially increases in those cells immediately surrounding the infection site. The data support the hypothesis that synthesis of PGIP constitutes an active deiense mechanism of plants that is elicited by signal molecules known to induce plant defense genes.

7. Augur, C., V. Stlefel, A. Darvill, P. Albersheim, and P. Puigdomenech. 1995. Molecular cloning and expression pattern of an $\alpha$-fucosidase gene from pea seedlings. J. Blol. Chem. 270: 24839-24843.

$\alpha-L-F u c o s i d a s e$ is a cell wall protein purified from pea (Pisum sativum) epicotyls. The $\alpha-L-$ fucosidase hydrolyzes terminal fucosyl residues from oligosaccharides of plant cell wall xyloglucan. $\alpha$-L-Fucosidase may be an important factor in plant growth regulation, as it inactivates fucose-containing xyloglucan oligosaccharides that inhibit growth of pea stem segments. The amino acid sequences of the $\mathrm{N}$-terminal region and one internal peptide were used to design redundant oligonucleotides that were utilized as primers in a PCR with CDNA generated from pea mRNA as the template. A specific PCR amplification product containing 357 base pairs was isolated, cloned, and sequenced. The deduced amino acid sequence included the two peptides used to design the primers for PCR plus two other peptides obtained by proteinase digestion of $\alpha$-L-fucosidase. No sequence homology to other $\alpha$-L-fucosidases was apparent, although the $\mathrm{N}$-terminal region is strongly homologous to Kunitz-type trypsin inhibitors. CDNA and genomic copies were isolated and sequenced. In pea, the gene is present in two or three copies. Its mRNA is present in roots, leaves, and elongating shoots. The spatial pattern of expression of the $\alpha$-L-fucosidase was determined by in situ hybridization.

8. Darvill, A., C. Bergmann, F. Cervone, G. De Lorenzo, K.-S. Ham, M.D. Spiro, W.S. York, and P. Albersheim. 1995. Oligosaccharins involved in plant growth and hostpathogen interactions. Biochem. Soc. Symp. 60: 89-94.

Carbohydrates, the building blocks of many structural polymers that give form to living cells and organisms, also play important roles in the interactions of cells with one another and with their environment. Plants and animals have evolved molecular signaling mechanisms to regulate the expression of genes essential for their growth, development, and defense against pests. Some of these signals or regulatory molecules are oligosaccharides. Oligosaccharides with regulatory activities are called oligosaccharins. The extensive stereochemistry, multiple hydroxyls and oxygen atoms, and accessible hydrophobic regions characteristic of glycosyl residues make oligosaccharides ideal ligands for specific interactions with recognition sites on proteins. These carbohydrate-binding proteins distinguish among the large number of primary structures and three-dimensional shapes which oligosaccharides can adopt. Thus, protein receptors can distinguish among a range of information-carrying oligosaccharides and transmit this information to the cells to which the receptors are attached. This paper describes recent progress we have made studying oligosaccharins active in plants. 
9. Wu, S.-C., S. Kauffmann, A.G. Darvill, and P. Albersheim. 1995. Purification, cloning and characterization of two xylanases from Magnaporthe grisea, the rice blast fungus. MPMI 8: 506-514.

Magnaporthe grisea, the fungal pathogen that causes rice blast disease, secretes two

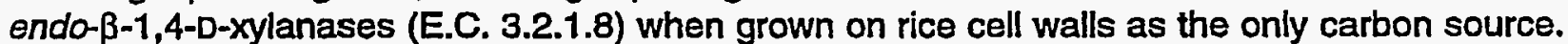
One of the xylanases, XYN33, is a 33-kD protein on sodium dodecyl sulfate-polyacrylamide gel and accounts for approximately $70 \%$ of the endoxylanase activity in the culture filtrate. The second xylanase, XYN22, is a 22-kD protein and accounts for approximately $30 \%$ of the xylanase activity. The two proteins were purified, cloned, and sequenced. XYN33 and XYN22 are both basic proteins with calculated isoelectric points of 9.95 and 9.71 , respectively. The amino acid sequences of XYN33 and XYN22 are not homologous, but they are similar, respectively, to family $F$ and family $G$ xylanases from other microorganisms. The genes encoding XYN33 and XYN22, designated $X Y N 33$ and $X Y N 22$, are single-copy in the haploid genome of $M$. grisea and are expressed when $M$. grisea is grown on rice cell walls or on oatspelt xylan, but not when grown on sucrose.

10. Ham, K.-S., P. Albersheim, and A.G. Darvill. 1995. Generation of $\beta$-glucan elicitors by plant enzymes and inhibition of the enzymes by a fungal proteln. Can J. Bot. 73(Suppl 1): S1100-S1103.

The biosynthesis and accumulation of phytoalexins is a well-studied plant defense response. Plants synthesize and accumulate phytoalexins in response to microbial infection or elicitor treatment. The $\beta$-glucan heptaglucoside is a well-studied phytoalexin elicitor isolated from partial acid hydrolysates of Phytophthora sojae f.sp. glycinea (Psg) mycelial walls. Using the soybean-Psg system, we have demonstrated that endo- $\beta-1,3-$ glucanases (EC 3.2.1.39) are the principal soybean enzymes involved in generating phytoalexin oligoglucoside elicitors from mycelial walls. We have also recently observed that Psg secretes a protein that inhibits the soybean endo- $\beta-1,3-$ glucanse activity that could release elicitors from fungal mycelial walls. The inhibitor protein, which has been purified to homogeneity, does not inhibit endo- $\beta-1,3-$ glucanases of the fungus or a tobacco pathogenesis-related endo- $\beta-1,3$-glucanase. The existence of the inhibitor protein in Psg suggests that pathogens have evolved specific proteins to inhibit the fungal wall-degrading enzymes of their host plants, just as plants have evolved proteins (e.g., pectic enzyme inhibitors) to inhibit plant cell wall-degrading enzymes secreted by their pathogens. It seems possible that pathogens secrete inhibitors of other pathogenesisrelated proteins (e.g., chitinases) and that the interplay of hydrolases and their inhibitors could determine the outcome of plant-pathogen interactions.

\section{Abstracts of Papers Submitted}

11. Stotz, H.U., C.W. Bergmann, P. Albersheim, A.G. Darvill, and J.M. Labavitch. 1995. Polygalacturonase inhibitor proteins from bean (Phaseolus vulgaris $L$ ), pear (Pyrus communis L) and tomato (Lycopersicon esculentum Mill.): Immunological relatedness and specificity of polygalacturonase inhibition. Submitted.

Purified polygalacturonase inhibitor proteins (PGIPs) from pods of bean (Phaseolus vulgaris L.) and from fruits of tomato (Lycopersicon esculentum Mill.) and pear (Pyrus communis L.) were tested for inhibition of polygalacturonases (PGs) from Aspergillus niger, Fusarium moniliforme and Botrytis cinerea. Bean PGIP equally inhibited all three fungal PGs. Tomato PGIP was less active against PGs from $A$. niger and $B$. cinerea than bean PGIP. Pear 
PGIP only inhibited PG from $B$. cinerea. Tomato PGIP, which noncompetitively inhibited $A$. niger PG, is kinetically more similar to bean (Lafitte et al., 1984, Physiol. Plant Pathol. 25: 3953) than pear PGIP (AbuGoukh et al., 1983, Physiol. Plant Pathol. 23: 111-122). Polyclonal antibodies raised against PGIPs from bean and pear were used to determine the immunological relatedness of the three PGIPs. Antibodies raised against deglycosylated pear PGIP crossreacted weakly with bean and tomato PGIPs, while antibodies raised against the bean glycoprotein cross-reacted weakly with pear and tomato PGIPs. Antibodies raised against an amino-terminal peptide from bean did not recognize pear PGIP. These data suggest that differences in the specific interaction of plant PGIPs with fungal PGs may be due to differences in PGIP structure.

12. Zablackis, E., W.S. York, M. Pauly, W.-D. Reiter, C.C.S. Chapple, P. Albersheim, and A. Darvill. 1995. L-Fucose is replaced by L-Galactose, a structural and conformational homologue, in the cell wall xyloglucan of Arabidopsis mutant murt. Submitted.

L-Fucose (6-deoxy-L-galactose) is a component of xyloglucans and other polysaccharides in the cell wall of most dicotyledonous plants, including Arabidopsis thaliana. Fucosylated xyloglucan oligosaccharides are signal molecules (oligosaccharins). We have purified and characterized xyloglucan oligosaccharides from the leaves of the fucose-deficient mur1 mutant $A$. thaliana and determined that in xyloglucan, L-fucose is replaced by its structural and conformational homologue, L-galactose. This modification does not detectably alter the biological activity of the xyloglucan oligosaccharins isolated from mur1. These results suggest that essential structural and conformational features of xyloglucan and xyloglucan-derived ollgosaccharins are retained when L-galactose replaces L-fucose, and that the availability of Lgalactose provides a plausible explanation for why murt is not a lethal mutation.

\section{Abstracts of Papers In Preparation}

13. Clay, R.P., C.W. Bergmann, and M.S. Fuller. 1995. Isolation and characterization of an endopolygalacturonase from Cochliobolus sativus and development of antibodies specific for the enzyme. In preparation.

Endopolygalacturonase of Cochliobolus sativus Ito and Kuribay (strain SB85) was produced in shake culture by growth of the organism on liquid medium containing citrus pectin as the sole carbon source. The endopolygalacturonase was recovered from the growth medium by alcohol precipitation and further purified by cation-exchange high performance liquid chromatography and size-exclusion filtration. The enzyme exhibits a weight of 33,977 daltons and an isoelectric point in the range of 9.0 to 9.5. The enzyme exhibited typical endo-activity as analyzed by viscometry. Activity of the enzyme was inhibited by the polygalacturonase inhibiting protein from Phaseolus vulgaris $\mathrm{L}$. (cultivar Pinto). The amino-terminus was sequenced by Edman degradation, with the derived amino acid sequence containing a 14amino acid region with homology to a region at the $N$-terminus of an endopolygalacturonase of C. carbonum Nelson. Monoclonal antibodies specific for the endopolygalacturonase were selected following immunization of mice with the native protein. Polyclonal antibodies were produced by immunization of rabbits with a multiple antigenic protein synthesized in accordance with the 14-amino acid homologous sequence. Western blot analysis confirmed specificity of the antibodies for the endopolygalacturonase of $C$. sativus. Western blot analysis detected endopolygalacturonase within a total protein profile from Hordeum vulgare L. (cultivar Golden Promise) leaf segments $18 \mathrm{~h}$ after inoculation with $c$. sativus spores. Indirect immunolocalization of pectin in barley epidermal cell walls was accomplished at the electron 
microscope level by exposing thin sections of the walls to purified $C$. sativus endopolygalacturonase, followed by a monoclonal antibody to the enzyme and finally antiantibody-gold.

14. Ham, K.-S., A.G. Darvill, and P. Albersheim. 1995. Fungal pathogens have a protection mechanism against plant pathogenesis-related endo- $\beta-1,3-g$ lucanases. In preparation.

Plant endo- $\beta-1,3-g$ lucanases and chitinases inhibit the growth of some fungi and generate elicitor-actlve oligosaccharides while depolymerizing polysaccharides of mycelial walls. Overexpression of the endo- $\beta-1,3$-glucanases and/or chitinases in transgenic plants provides, in some but not all cases, increased protection against fungal pathogens. Indeed, most of the phytopathogenic fungi tested in vitro are resistant to endo- $\beta-1,3-g l u c a n a s e s$ and chitinases. Some phytopathogenic fungi whose growth is inhibited by these enzymes are able to overcome, over a period of hours, the effect of these enzymes, indicating an ability of those fungi to adapt to the enzymes. We now present evidence that fungal pathogens secrete a set of proteins that inhibit, with a high degree of selectivity, individual plant endo- $\beta-1,3-$ glucanases. We have purified to homogeneity a glucanase inhibitor protein (GIP-1) from the culture fluid of the fungal pathogen of soybeans, Phytophthora sojae f.sp. glycinea (Psg). We have also purified, from soybean seedlings, two basic pathogenesis-related endo- $\beta-1,3-$ glucanases (EnGLsoy-A and EnGLsoy-B). GIP-1 inhibits EnGLsoy-A but not EnGLsoy-B. Moreover, GIP-1 does not inhibit endo- $\beta-1,3-$ glucanases secreted by Psg itself nor does GIP-1 inhibit PR-O, a pathogenesis-related endo- $\beta-1,3$-glucanase of tobacco which is not a host of Psg. We present evidence that Psg secretes other GIPs that inhibit other endo- $\beta-1,3$-glucanase(s) of soybean. These results demonstrate specific interactions between individual gene products of the host and pathogen and establish the need to consider fungal proteins that inhibit plant endo- $\beta-1,3-$ glucanases (and presumably chitinases) when attempting to use the genes encoding endo- $\beta$ 1,3-glucanases and chitinases to engineer resistance to fungi in transgenic plants.

15. Ham, K.-S., P. Albersheim, and A.G. Darvill. 1995. Plant enzymes generate oligoglucoside phytoalexin elicitors from the mycelial walls of fungi. In preparation.

Phytoalexin accumulation is a well-studied plant defense response. Phytoalexins are low molecular weight antibiotics that are synthesized by and accumulate in plant cells in response to microbial infection. Accumulation of phytoalexins can also be induced when plants are exposed to elicitors of both plant and pathogen origin. Fungal $\beta$-glucan elicitors have been studied extensively. These elicitors were first detected in the culture filtrate and later in cell wall preparations of a fungal pathogen of soybean, Phytophthora sojae f.sp. glycinea (Psg). The smallest elicitor-active oligo- $\beta$-glucoside was isolated from a partial acid hyrolyzate of Psg mycelial walls and identified as a hepta- $\beta$-glucoside. It was found that the structural requirements for biological activity of the elicitor-active hepta- $\beta$-glucoside (AHG) are precise and that the reducing terminal glycosyl residue of AHG is not essential for elicitor activity, indicating that the hexa- $\beta$-glucoside portion (the AHG minus the reducing terminal glucosyl residue) of the elicitor is the minimum structural element for AHG to be active. As AHG was released from fungal cell wall glucans by partial acid hydrolysis, it remains to be determined whether the AHG (or hexa- $\beta$-glucoside) elicitor can be generated from mycelial walls by enzymes present in infected plants. 
16. Spiro, M.D., B.L. Ridley, J. Glushka, A.G. Darvill, and P. Albersheim. 1995. Synthesis and characterization of tyramine-derivatized 1,4-linked $\alpha-D-$ oligogalacturonides. In preparation.

The reducing end $C-1$ of 1,4 -linked $\alpha-D$-oligogalacturonides (oligogalacturonides), with degrees of polymerization of 3 and 13, was coupled to tyramine via reductive amination in the presence of sodium cyanoborohydride. These derivatives were purified in milligram quantities and structurally characterized. Tyramination of trigalacturonic acid proceeded to completion. The yield of apparently homogeneous tyraminated trigalacturonic acid after desalting was $35 \%$. Derivatization of tridecagalacturonide with tyramine was incomplete. The tyraminated tridecagalacturonide was purfied to apparent homogeneity using semi-preparative highperformance anion-exchange chromatography (HPAEC) with a yield of $30 \%$. The structures of the derivatized ollgogalacturonides were established by ${ }^{1} \mathrm{H}$ NMR spectroscopy and electrospray mass spectrometry. 